\title{
Photonic Material Selection of Scintillation Crystals Using Monte Carlo Method for X-Ray Detection in Industrial Computed Tomography
}

\author{
Peng He, Biao Wei, Mi Zhou, Peng Feng, and Mianyi Chen \\ The Key Lab of Optoelectronic Technology and Systems of the Education Ministry of China, \\ Chongqing University, Chongqing 400044, China
}

Correspondence should be addressed to Biao Wei; weibiao@cqu.edu.cn

Received 26 March 2014; Accepted 26 April 2014; Published 21 May 2014

Academic Editor: Mohd Hafiz Fazalul Rahiman

Copyright (C) 2014 Peng He et al. This is an open access article distributed under the Creative Commons Attribution License, which permits unrestricted use, distribution, and reproduction in any medium, provided the original work is properly cited.

Currently industrial X-CT system is designed according to characteristics of test objects, and test objects determine industrial $\mathrm{X}$-CT system structure, X-ray detector/sensor property, scanning mode, and so forth. So there are no uniform standards for the geometry size of scintillation crystals of detector. Moreover, scintillation crystals are usually mixed with some highly toxic impurity elements, such as $\mathrm{Tl}$ and $\mathrm{Cd}$. Thus, it is indispensable for establishing guidelines of engineering practice to simulate X-ray detection performances of different scintillation crystals. This paper focuses on how to achieve high efficient X-ray detection in industrial XCT system which used Monte Carlo (MC) method to study X-ray energy straggling characteristics, full energy peak efficiency, and conversion efficiency of some scintillation crystals (e.g., $\mathrm{CsI}(\mathrm{Tl}), \mathrm{NaI}(\mathrm{Tl})$, and $\mathrm{CdWO}_{4}$ ) after X-ray interacted with these scintillation crystals. Our experimental results demonstrate that $\mathrm{CsI}(\mathrm{Tl})$ scintillation crystal has the advantages of conversion efficiency, spectral matching, manufacturing process, and full energy peak efficiency; it is an ideal choice for high efficient X-ray detection in industrial X-CT system.

\section{Introduction}

Industrial X-ray computed tomography (X-CT) system is an advanced nondestructive measurement technique; it has been widely used in aerospace, weapons, metallurgy, machinery, electric power, geology, and so forth [1-5]. X-ray detector/sensor is an important component of industrial XCT system, which determines X-ray detection efficiency in data acquisition. CT image quality relies on X-ray detection efficiency [6]; thus how to achieve the high efficient X-ray detection is a key technique in industrial X-CT research field.

In current industrial X-CT system, X-ray detection method is transforming X-ray photons into visible light photons via scintillation crystals or screens (e.g., CsI(Tl), $\mathrm{NaI}(\mathrm{Tl})$, and $\mathrm{CdWO}_{4}$ ) [7-9] at first and then converting light signal into electrical signal by means of photoelectric conversion devices (e.g., photomultiplier tube (PMT), photodiode (PD), and charge coupled device (CCD)) [10-13]. In order to improve resolution and signal to noise ratio (SNR) of CT images, it is necessary to select proper thickness of scintillation crystal [14-16]. If the thickness of scintillation crystal is too thick, it will affect the resolution of CT images. Meanwhile, if the thickness of scintillation crystal is too thin, it will reduce the luminous efficiency of scintillation crystal, which determines the SNR of CT images.

How to select proper thickness of scintillation crystal depends on the design of industrial X-CT system. Generally, the design of industrial X-CT system is determined by test objects, and the diameter range of test objects is from several millimeters to several hundred millimeters; the density range of test objects is from several $\mathrm{kg} / \mathrm{m}^{3}$ to several hundred $\mathrm{kg} / \mathrm{m}^{3}$. Thus, the X-ray energy and detection range are different for different industrial X-CT systems; it is difficult to make a uniform standard for the geometry size of scintillation crystals. Different scintillation crystals have different properties of X-ray detection, and some scintillation crystals have been 


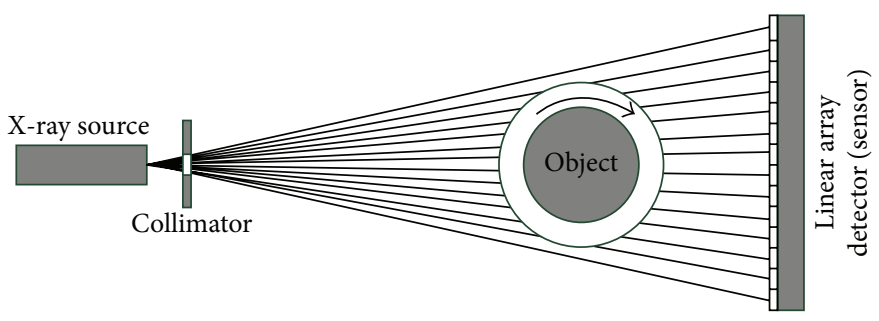

FIGURE 1: A typical X-ray detection model based on scintillation crystals in industrial X-CT system.

mixed with impurity elements (thallium (Tl) or cadmium (Cd)) which are harmful to people's health, especially $\mathrm{CsI}(\mathrm{Tl})$, $\mathrm{NaI}(\mathrm{Tl})$, and $\mathrm{CdWO}_{4}$.

In order to study how to keep enough X-ray energy deposition in $\mathrm{CsI}(\mathrm{Tl}), \mathrm{NaI}(\mathrm{Tl})$, and $\mathrm{CdWO}_{4}$ scintillation crystals and transform more X-ray photons into visible light photons to obtain high spatial resolution of CT images in industrial X-CT system, it is a reasonable scheme to simulate the photonic properties of $\mathrm{CsI}(\mathrm{Tl}), \mathrm{NaI}(\mathrm{Tl})$, and $\mathrm{CdWO}_{4}$ scintillation crystals using Monte Carlo (MC) method [1726]. Monte Carlo (MC) methods are stochastic techniques, which can be used in nuclear physics to analyze interaction between X-ray photons and scintillation crystals.

\section{Materials and Methods}

There are several X-ray detection modes in industrial X-CT system according to different data capture patterns, which mainly include linear array detector and area array detector. Generally, the X-ray detection mode based on CsI(Tl), $\mathrm{NaI}(\mathrm{Tl})$, or $\mathrm{CdWO}_{4}$ scintillation crystals adopts linear array detector or area array detector in industrial X-CT system [1013], and a typical X-ray detection model is shown in Figure 1, which mainly contains X-ray source, collimator, scintillation crystal detector, and so forth.

When X-ray photons and scintillation crystals interact with each other, due to photoelectric effect, Compton scattering, and electron pair effect, X-ray attenuation characteristics can be expressed as

$$
\mu=\rho\left(\frac{a Z^{3.8}}{E^{3.2}}+f_{\mathrm{KN}}(E)\right)
$$

where $\mu$ is X-ray linear attenuation coefficient, $\rho$ is the electron density, $a$ is a fitting parameter, $Z$ is the effective atomic number, $E$ is X-ray photon energy, and $f_{\mathrm{KN}}(E)$ is the Klein-Nishina function which yields the electronic cross section of Compton scattering. Finally X-ray photons are transformed into visible light photons detected by back-end optoelectronic devices. When the X-ray photon energy is lower than $1 \mathrm{MeV}$, it will be easy that photoelectric effect and Compton scattering occur. If photoelectric effect occurs, the optoelectronic device will record collective charges directly. If Compton scattering occurs, scatting photons will be calculated to compensate incident photons at first and then the optoelectronic device will record collective charges

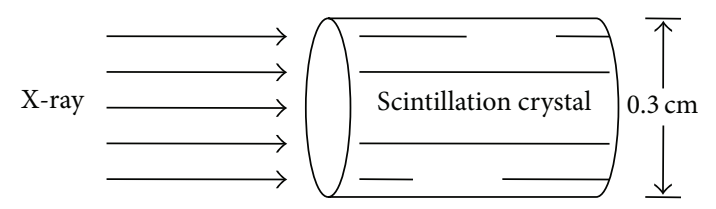

Figure 2: The Monte Carlo simulation model in our study.

as incident photons. The interaction patterns between $\mathrm{X}$ ray photons and scintillation crystals are random, but the occurrence probability depends on interaction cross section.

Generally, the scintillation crystal used in array detector of industrial X-CT system adopts cylinder design [27, 28]. In our Monte Carlo simulation study, we assume that the energy of X-ray source is monochromatic and adjustable, the X-ray beam is a parallel beam after X-ray passes through the front collimator, the diameter of scintillation crystal is $0.3 \mathrm{~cm}$, and the length of cylinder could be changed, as shown in Figure 2. Then we studied photonic properties of $\mathrm{CsI}(\mathrm{Tl}), \mathrm{NaI}(\mathrm{Tl})$, and $\mathrm{CdWO}_{4}$ scintillation crystals which interacted with different energy X-ray photons, and we assume that detector to object distance is $100 \mathrm{~cm}$, and $\mathrm{X}$-ray source to object distance is also $100 \mathrm{~cm}$ in our simulation. In our study, the number of photons produced by the X-ray source is 5 million; the cutoff condition of photons includes energy cut-off and position cut-off, which means in our simulation program we will not track these particles if the energy of photons is less than $0.001 \mathrm{MeV}$, and we will also not track these particles if photons escape from scintillation crystal. The cut-off condition of fluorescent photons is the length cut-off, which means in our simulation program we will cut this fluorescent beam if fluorescent transmission distance is more than $150 \mathrm{~mm}$.

\section{Results and Discussions}

In our simulation, we focus on photonic properties of scintillation detector. It is essential to design the physics and mathematics model of X-ray detection at first, and then we utilize a Monte Carlo software (EGS) to analyze characteristics of interaction between X-ray photons and scintillation crystals [17-26]. In this paper, we study three scintillation crystals $\mathrm{CsI}(\mathrm{Tl}), \mathrm{NaI}(\mathrm{Tl})$, and $\mathrm{CdWO}_{4}$; the physical properties of the three scintillation crystals are shown in Table 1.

3.1. Energy Straggling Characteristics. We simulated X-ray energy straggling characteristics after the three scintillation 


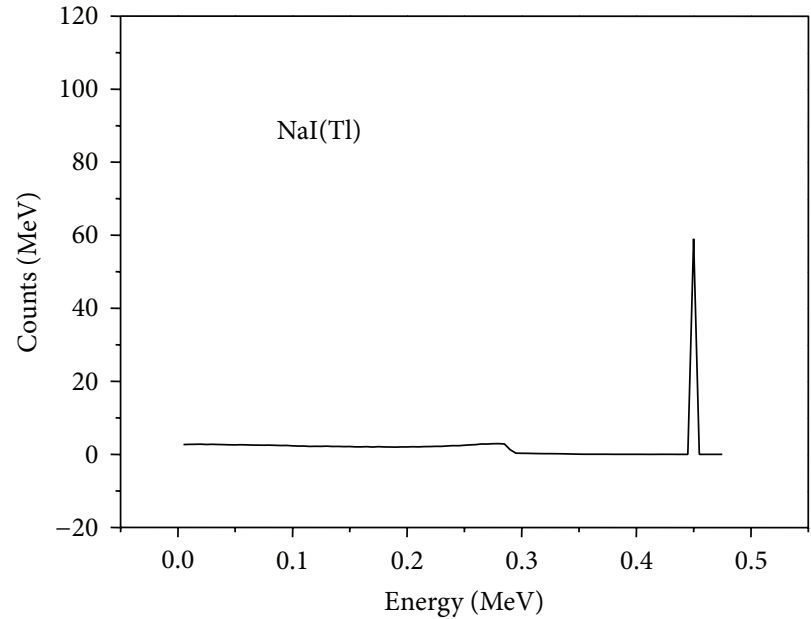

(a)

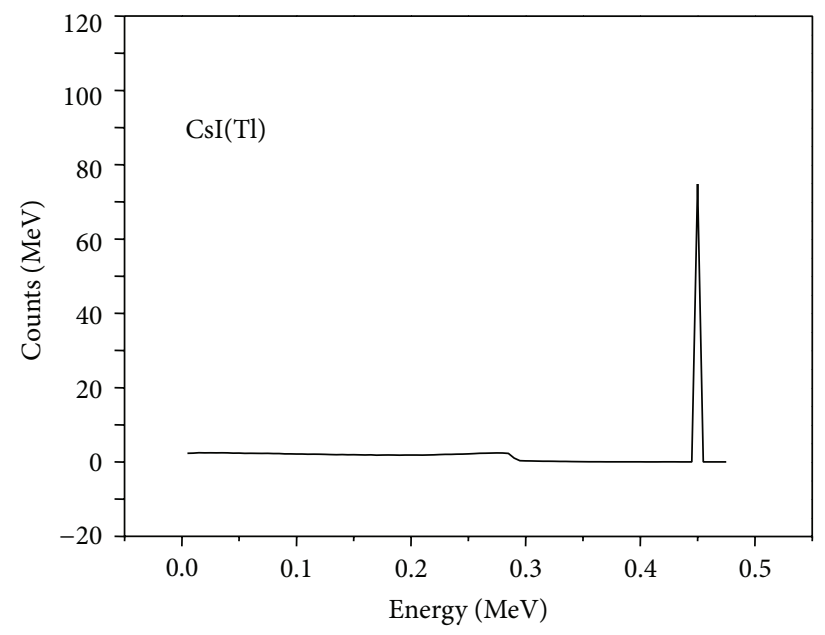

(b)

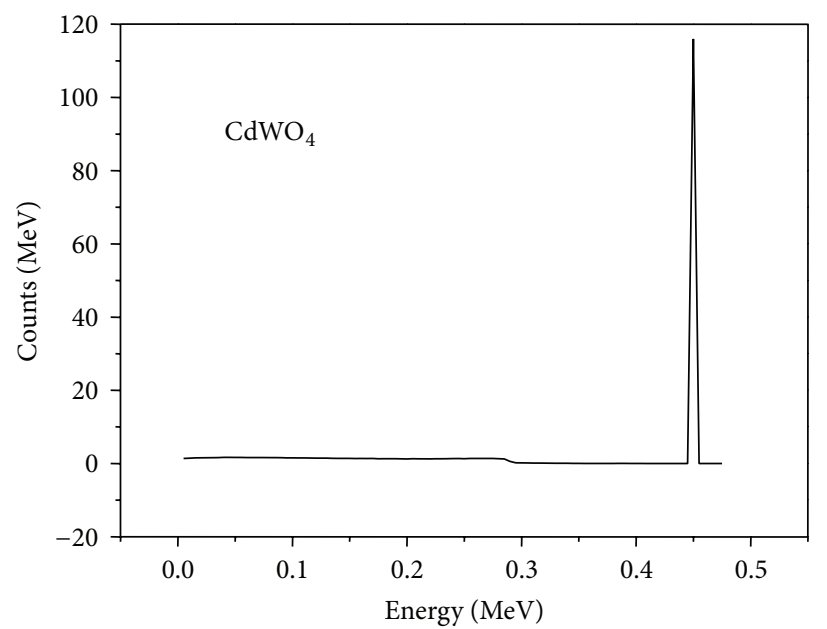

(c)

FIGURE 3: The simulation results of X-ray energy straggling characteristics after the three scintillation crystals interacted with X-ray incident photons.

TABLE 1: The physical properties of the three scintillation crystals.

\begin{tabular}{lccc}
\hline Items & $\mathrm{CsI}(\mathrm{Tl})$ & $\mathrm{NaI}(\mathrm{Tl})$ & $\mathrm{CdWO}_{4}$ \\
\hline Density & $4.53 \mathrm{~g} / \mathrm{cm}^{3}$ & $3.67 \mathrm{~g} / \mathrm{cm}^{3}$ & $7.99 \mathrm{~g} / \mathrm{cm}^{3}$ \\
Atomic number & 54 & 50 & 64.2 \\
Tl concentration & $0.1 \%$ & $0.1 \%$ & $/$ \\
Refractive index & 1.79 & 1.85 & 2.3 \\
Peak wavelength & $560 \mathrm{~nm}$ & $410 \mathrm{~nm}$ & $540 \mathrm{~nm}$ \\
\hline
\end{tabular}

crystals $\mathrm{CsI}(\mathrm{Tl}), \mathrm{NaI}(\mathrm{Tl})$, and $\mathrm{CdWO}_{4}$ interacted with X-ray incident photons. We assume that the X-ray incident photon energy is $450 \mathrm{KeV}$ and the length of scintillation crystal is $1.5 \mathrm{~cm}$. The simulation results of $\mathrm{X}$-ray energy straggling characteristics are shown in Figure 3; from Figure 3 we can see that X-ray energy straggling characteristics are similar after the three scintillation crystals interacted with X-ray incident photons, and the number of survival $450 \mathrm{KeV}$ photons is much more than other energy photon numbers. However, the three scintillation crystals $\mathrm{CsI}(\mathrm{Tl}), \mathrm{NaI}(\mathrm{Tl})$, and $\mathrm{CdWO}_{4}$ have different photonic properties; after $450 \mathrm{KeV} \mathrm{X}$-ray incident photons interacted with $\mathrm{CsI}(\mathrm{Tl}), \mathrm{NaI}(\mathrm{Tl})$, and $\mathrm{CdWO}_{4}$, the number of survival $450 \mathrm{KeV}$ photons which interacted with $\mathrm{CdWO}_{4}$ is much more than numbers of survival $450 \mathrm{KeV}$ photons which interacted with the other two scintillation crystals (CsI(Tl) and $\mathrm{NaI}(\mathrm{Tl})$ ), and the number of survival $450 \mathrm{KeV}$ photons which interacted with $\mathrm{CsI}(\mathrm{Tl})$ is more than the number of survival $450 \mathrm{KeV}$ photons which interacted with $\mathrm{NaI}(\mathrm{Tl})$.

3.2. Full-Energy Peak Efficiency. In Monte Carlo simulation study of full-energy peak efficiency, we assume that the lengths of $\mathrm{CsI}(\mathrm{Tl}), \mathrm{NaI}(\mathrm{Tl})$, and $\mathrm{CdWO}_{4}$ scintillation crystals are $0.3 \mathrm{~cm}, 0.5 \mathrm{~cm}, 1.0 \mathrm{~cm}$, and $1.5 \mathrm{~cm}$, respectively, and the Xray source energies are $120 \mathrm{KeV}, 160 \mathrm{KeV}, 220 \mathrm{KeV}, 320 \mathrm{KeV}$, 


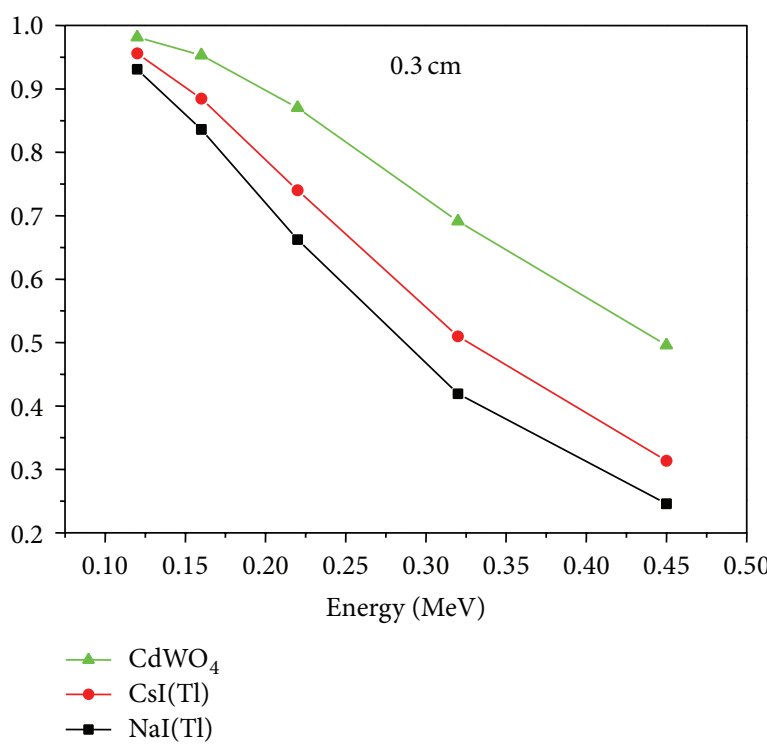

(a)

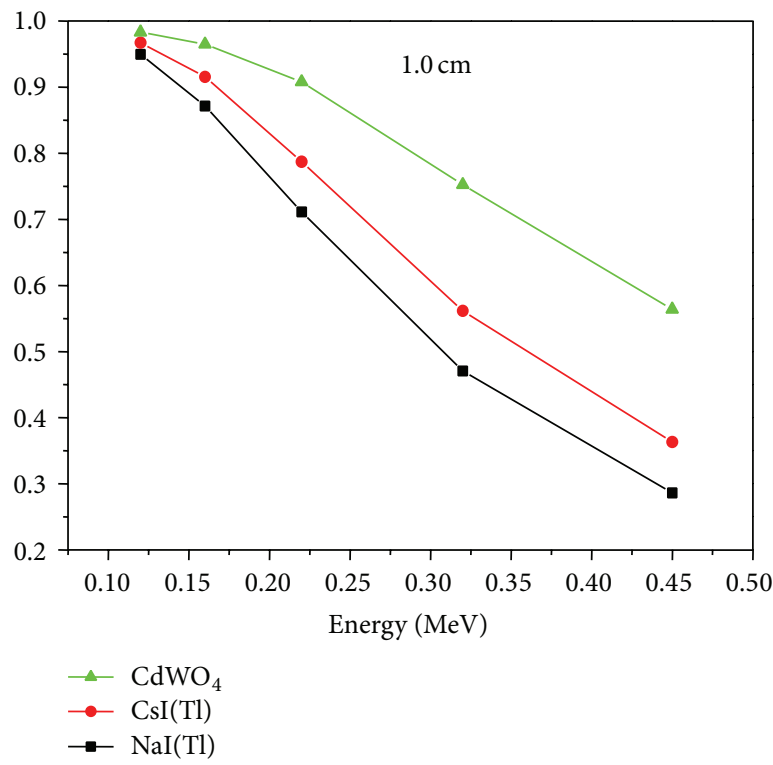

(c)

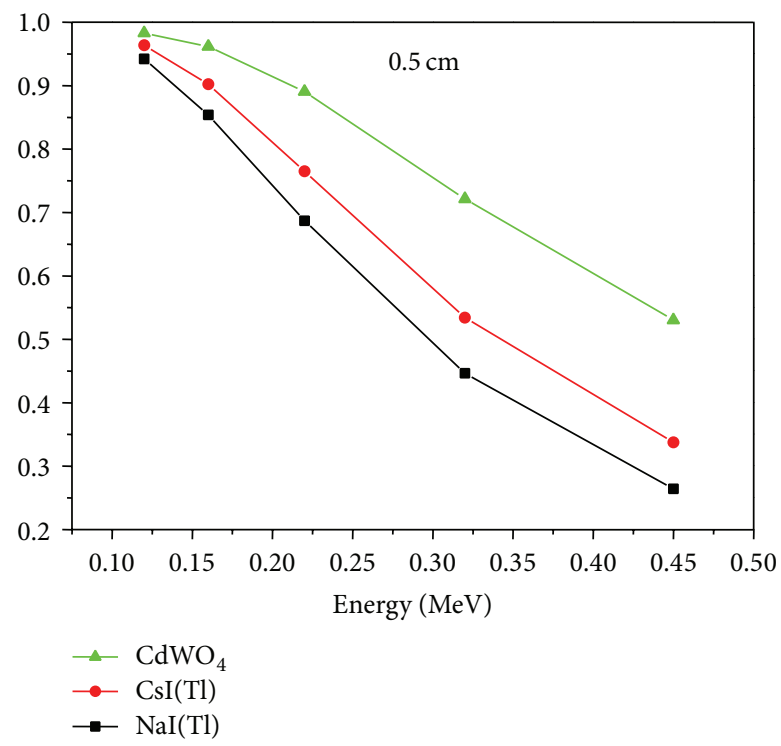

(b)

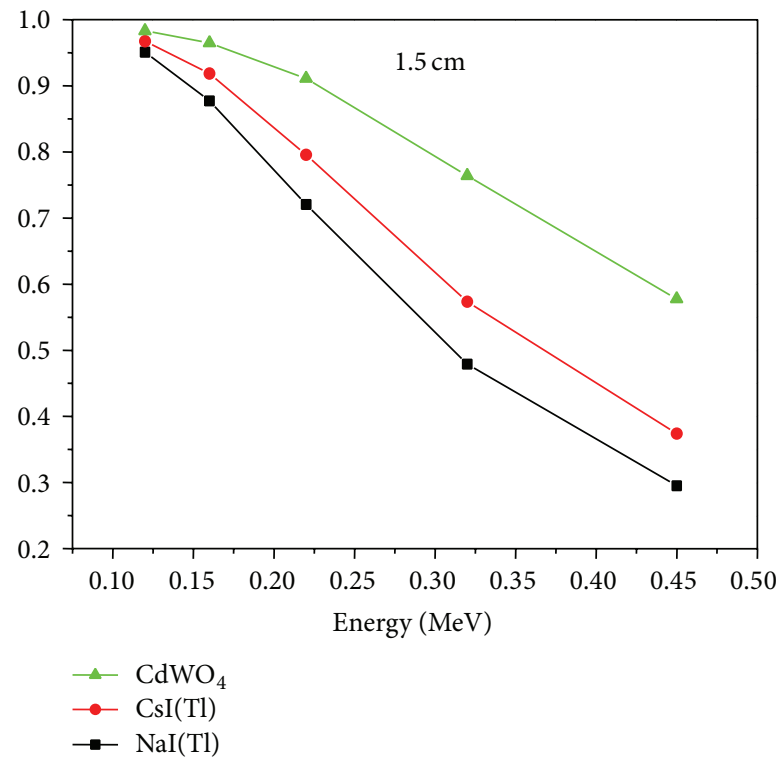

(d)

FIGURE 4: The simulation results of full-energy peak efficiencies of three scintillation detectors with different lengths.

and $450 \mathrm{KeV}$, respectively; Figure 4 shows the simulation results of full-energy peak efficiency.

From Figure 4, we can see that full-energy peak efficiencies of the three scintillation detectors decrease rapidly with the X-ray energy increasing. When energies of X-ray incident photons which interacted with the three scintillation detectors are the same, the $\mathrm{CdWO}_{4}$ scintillation detector has the highest full-energy peak efficiency, and $\mathrm{NaI}(\mathrm{Tl})$ scintillation detector has the lowest full-energy peak efficiency. The three scintillation detectors have different full-energy peak efficiencies due to the different physical properties of three scintillation crystals. In particular, after $\mathrm{CsI}(\mathrm{Tl})$ crystal interacted with X-ray photons, the peak wavelength of visible emission spectrum is $540 \mathrm{~nm}$, it is easy to match spectral response of CCD devices, which is benefit for improving SNR of CT image.

3.3. Scintillation Crystal Conversion Efficiency. To simulate the three scintillation crystal conversion efficiencies using Monte Carlo method, we assume that the lengths of $\mathrm{CsI}(\mathrm{Tl})$, $\mathrm{NaI}(\mathrm{Tl})$, and $\mathrm{CdWO}_{4}$ scintillation crystals are $0.3 \mathrm{~cm}, 0.5 \mathrm{~cm}$, $1.0 \mathrm{~cm}$, and $1.5 \mathrm{~cm}$, respectively, and the X-ray source energies are $120 \mathrm{KeV}, 160 \mathrm{KeV}, 220 \mathrm{KeV}$, $320 \mathrm{KeV}$, and $450 \mathrm{KeV}$, respectively. The simulation results are shown in Figure 5. 


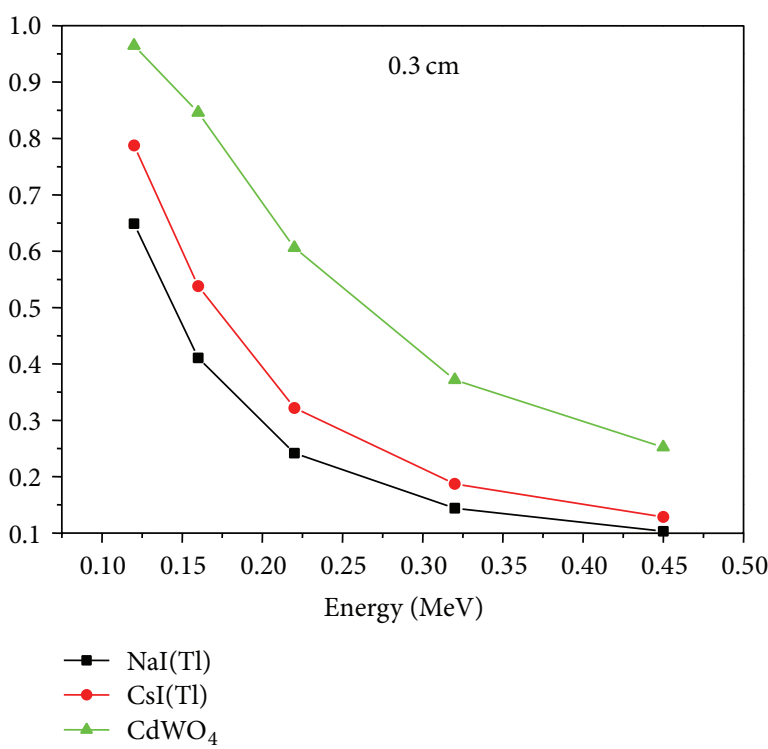

(a)

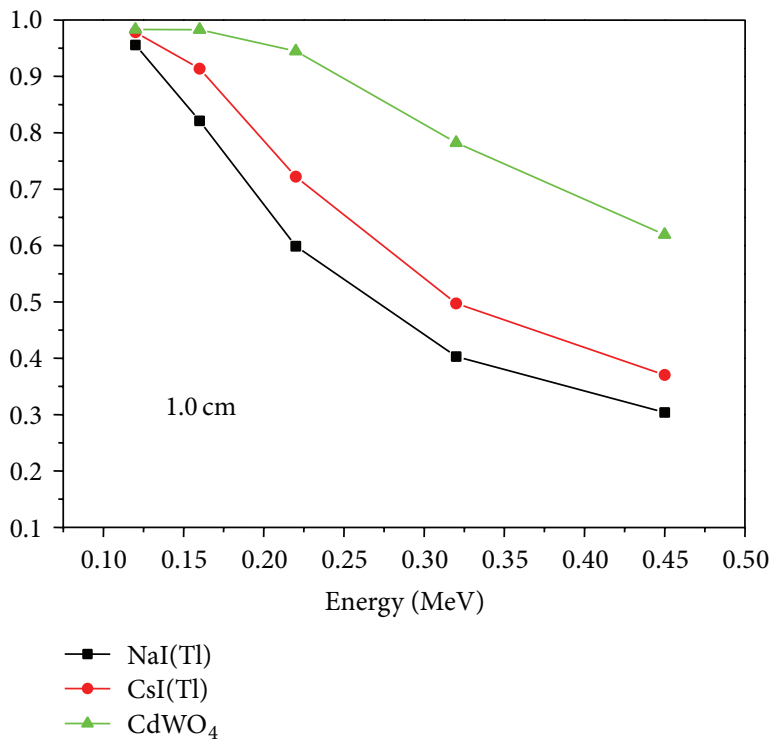

(c)

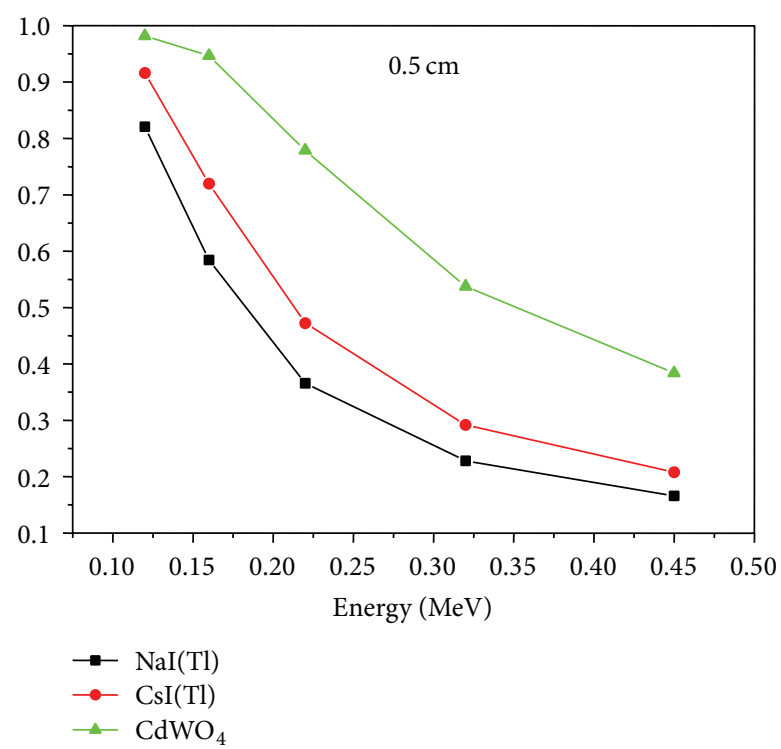

(b)

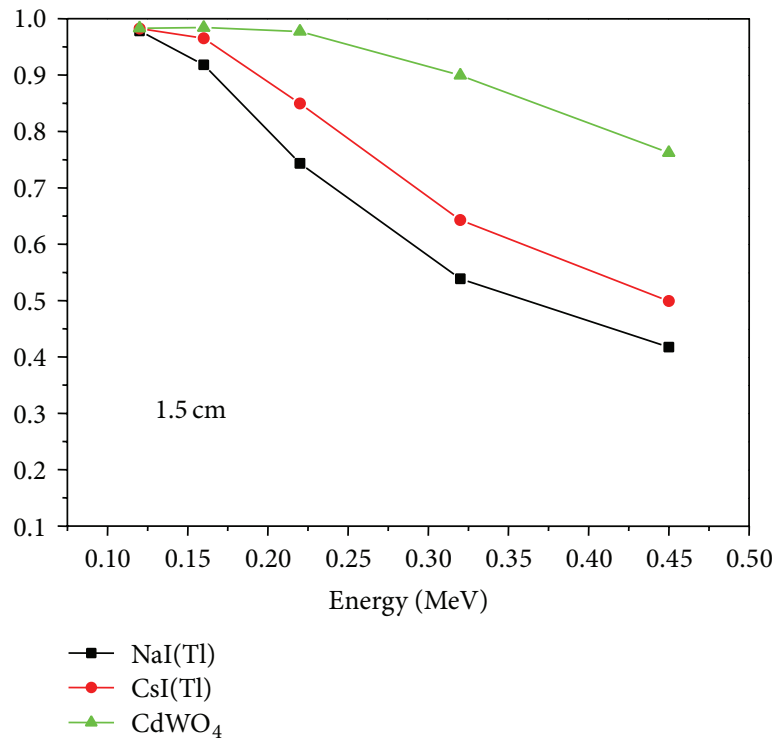

(d)

FIGURE 5: The simulation results of conversion efficiencies of the three scintillation crystals $\mathrm{CsI}(\mathrm{Tl}), \mathrm{NaI}(\mathrm{Tl}), \mathrm{and}_{\mathrm{CdWO}}$.

From Figure 5, we can see that the conversion efficiencies of the three scintillation crystals decrease gradually with Xray photon energy increasing regardless of scintillation crystal type and length, and $\mathrm{CdWO}_{4}$ has the highest conversion efficiency. When the length of the crystal is $1.0 \mathrm{~cm}$ or $1.5 \mathrm{~cm}$ and the $\mathrm{X}$-ray photon energy is $120 \mathrm{KeV}$, the conversion efficiency of $\mathrm{CdWO}_{4}$ could reach almost $100 \%$. Interacted with the same energy incident photons, the conversion efficiency of $\mathrm{CsI}(\mathrm{Tl})$ is lower than that of $\mathrm{CdWO}_{4}$, and the conversion efficiency of $\mathrm{NaI}(\mathrm{Tl})$ is the lowest. However, the refractive index of $\mathrm{CdWO}_{4}$ scintillation crystal is only 2.3 which affects visible light transmission in scintillation crystals, and the average wavelength of visible emission spectrum is $480 \mathrm{~nm}$ which is not easy to match spectral response of CCD devices.

\section{Conclusions}

In industrial X-CT system, it is necessary to make personalized design due to different test objects. There are no uniform standards for the geometry size of scintillation crystals, and the crystals are mixed with some highly toxic impurity elements, such as $\mathrm{Tl}$ and $\mathrm{Cd}$. Thus, it is indispensable for establishing guidelines of engineering practice to simulate $\mathrm{X}$ ray detection performances of different scintillation crystals using Monte Carlo method.

In this paper, we study X-ray detection performances of scintillation crystals $\mathrm{CsI}(\mathrm{Tl}), \mathrm{NaI}(\mathrm{Tl})$, and $\mathrm{CdWO}_{4}$ using Monte Carlo method; the experimental results demonstrated that $\mathrm{CdWO}_{4}$ scintillation crystals show good performances 
in full-energy peak efficiency and scintillation conversion efficiency, which could be widely used in high-energy (above $2 \mathrm{MeV}$ ) X-ray detection of the industrial X-CT system. $\mathrm{NaI}(\mathrm{Tl})$ scintillation crystal has lower full-energy peak efficiency and scintillation conversion efficiency, and it is easily deliquesced, so $\mathrm{NaI}(\mathrm{Tl})$ scintillation crystal is unsuitable for $\mathrm{X}$-ray detection in industrial X-CT system. The full-energy peak efficiency and conversion efficiency of $\mathrm{CsI}(\mathrm{Tl})$ crystal are better than those of $\mathrm{NaI}(\mathrm{Tl})$ scintillation crystal, and CsI(Tl) scintillation crystal has the advantage of nondeliquescence and manufacturing process, which is a benefit for X-ray detection and detector structure design in industrial X-CT system. In our simulation study, CsI(Tl) scintillation crystal adopts cylinder design, whose diameter is $0.3 \mathrm{~cm}$. When the length of $\mathrm{CsI}(\mathrm{Tl})$ scintillation crystal is $1.5 \mathrm{~cm}$ and X-ray photon energy is $220 \mathrm{KeV}$, the full-energy peak efficiency can reach $79.6 \%$ and the conversion efficiency can reach $85 \%$. Thus, $\mathrm{CsI}(\mathrm{Tl})$ as a scintillation crystal is an ideal choice for high efficient X-ray detection in industrial X-CT system.

\section{Conflict of Interests}

The authors declare that there is no conflict of interests regarding the publication of this paper.

\section{Acknowledgments}

This work was supported by the National Natural Science Foundation of China (no. 61171157 and no. 61201346) and the Fundamental Research Funds for the Central Universities (no. CDJZR14125501).

\section{References}

[1] K. A. Spencer, "Computer tomography-an overview," The Journal of Photographic Science, vol. 37, no. 2, pp. 84-89, 1989.

[2] M. V. Reddy, S. N. Lukose, M. P. Subramanian, G. V. Siva Rao, C. Muralidhar, and K. Balasubramaniam, "Industrial computed tomography system for aerospace applications: development and characterization," Insight: Non-Destructive Testing and Condition Monitoring, vol. 53, no. 6, pp. 307-311, 2011.

[3] P. Schuetz, A. Miceli, I. Jerjen et al., "Reducing environmental scattering in industrial computed tomography by system redesign," NDT \& E International, vol. 58, pp. 36-42, 2013.

[4] W. Dewulf, K. Kiekens, Y. Tan, F. Welkenhuyzen, and J. P. Kruth, "Uncertainty determination and quantification for dimensional measurements with industrial computed tomography," CIRP Annals: Manufacturing Technology, vol. 62, no. 1, pp. 535-538, 2013.

[5] T. Luthi, A. Flisch, and P. Wyss, "Industrial computed X-ray tomography," Insight: Non-Destructive Testing and Condition Monitoring, vol. 40, no. 3, pp. 196-197, 1998.

[6] Z. Guo, Z. W. Tang, X. Z. Wang, M. L. Deng, G. S. Hu, and H. Zhang, "Performance evaluation of a modular detector unit for X-ray computed tomography," Sensors, vol. 13, no. 4, pp. 51675180, 2013.

[7] E. Sakai, "Recent measurements on scintillator-photodetector systems," IEEE Transactions on Nuclear Science, vol. 34, no. 1, pp. 418-422, 1986.
[8] B. C. Grabmaier, "Crystal scintillators," IEEE Transactions on Nuclear Science, vol. 31, no. 1, pp. 372-376, 1983.

[9] W. W. Moses, "Current trends in scintillator detectors and materials," Nuclear Instruments and Methods in Physics Research A: Accelerators, Spectrometers, Detectors and Associated Equipment, vol. 487, no. 1-2, pp. 123-128, 2002.

[10] R. Clarke, "CCD X-ray detectors: opportunities and challenges," Nuclear Instruments and Methods in Physics Research A: Accelerators, Spectrometers, Detectors and Associated Equipment, vol. 347, no. 1-3, pp. 529-533, 1994.

[11] "Industrial 3D Computed Tomography (CT) X-Ray Systems," http://www.xviewct.com/.

[12] S. Izumi, S. Kamate, K. Satoh, and H. Miyai, "High energy xray computed tomography for industrial applications," IEEE Transactions on Nuclear Science, vol. 40, no. 2, pp. 158-161, 1993.

[13] S. Kasap, J. B. Frey, G. Belev et al., "Amorphous and polycrystalline photoconductors for direct conversion flat panel x-ray image sensors," Sensors, vol. 11, no. 5, pp. 5112-5157, 2011.

[14] J. S. Karp and M. E. Daube-Witherspoon, "Depth-of-interaction determination in $\mathrm{NaI}(\mathrm{Tl})$ and BGO scintillation crystals using a temperature gradient," Nuclear Instruments and Methods in Physics Research A: Accelerators, Spectrometers, Detectors and Associated Equipment, vol. 260, no. 2-3, pp. 509-517, 1987.

[15] A. Saoudi, C. M. Pepin, F. Dion et al., "Investigation of depth-ofinteraction by pulse shape discrimination in multicrystal detectors read out by avalanche photodiodes," IEEE Transactions on Nuclear Science, vol. 46, no. 3, pp. 462-467, 1999.

[16] E. J. Morton, W. Swindell, D. G. Lewis, and P. M. Evans, "A linear array, scintillation crystal-photodiode detector for megavoltage imaging," Medical Physics, vol. 18, no. 4, pp. 681-691, 1991.

[17] A. F. Bielajew, H. Hirayama, W. R. Nelson, and D. W. O. Rogers, "History, overview and recent improvements of EGS4," Tech. Rep. NRC-PIRS-0436, National Research council of Canada, 1994.

[18] J. Chavanelle, A. Pousse, L. Fagot, M. Parmentier, and B. Kastler, "Scintillator crystal optimization by Monte Carlo simulation for photodiode matrix detector," in Proceedings of the 2000 IEEE Nuclear Science Symposium Conference Record, vol. 3, pp. 16-19, October 2000

[19] H.-H. Hsu and H. Lee, "Monte Carlo calculations of the vacuum Compton detector sensitivities," IEEE Transactions on Nuclear Science, vol. 36, no. 6, pp. 1926-1930, 1989.

[20] F. Vittori, F. de Notaristefani, T. Malatesta, and D. Puertolas, "A study on light collection of small scintillating crystals," Nuclear Instruments and Methods in Physics Research A: Accelerators, Spectrometers, Detectors and Associated Equipment, vol. 452, no. 1-2, pp. 245-251, 2000.

[21] G. Tzanakos and T. Thireou, "A simulation study of the performance characteristics of a YAP: Ce detector for positron emission tomography," in Proceeding of the 20th Annual International Conference of the IEEE Engineering in Medicine and Biology Society, vol. 2, pp. 755-758, Hong Kong, 1998.

[22] S. A. Pavlopoulos and T. Thireou, "Monte Carlo simulation of a YAP: Ce block detector for high resolution positron emission tomography," in Proceeding of the 20th Annual International Conference of the IEEE Engineering in Medicine and Biology Society, vol. 2, pp. 752-754, Hong Kong, 1998.

[23] J. Michael, S. M. Flynn, S. J. Wilderman, and J. J. Ciarelli, "Quantum noise in digital X-ray image detectors with optically coupled scintillations," IEEE Transactions on Nuclear Science, vol. 43, no. 4, pp. 2320-2325, 1996. 
[24] S.-J. Ren, W.-B. Sang, W. Jin, W.-W. Li, Q. Zhang, and J.-H. Min, "Primary study of Monte Carlo simulation on CdZnTe nuclear detector," High Energy Physics and Nuclear Physics, vol. 28, no. 2, pp. 191-195, 2004.

[25] S. Kerisit, K. M. Rosso, and B. D. Cannon, "Kinetic Monte Carlo model of scintillation mechanisms in CsI and CsI(Tl)," IEEE Transactions on Nuclear Science, vol. 55, no. 3, pp. 1251-1258, 2008.

[26] N. Ghazanfari, S. Sarkar, G. Loudos, and M. R. Ay, "Quantitative assessment of crystal material and size on the performance of rotating dual head small animal PET scanners using Monte Carlo modeling," Hellenic Journal of Nuclear Medicine, vol. 15, no. 1, pp. 33-39, 2012.

[27] J. G. Park, S. H. Jung, J. B. Kim, J. Moona, and C. H. Kimb, "Influence of void on image quality of industrial SPECT," Journal of Instrumentation, vol. 8, Article ID P12011, 2013.

[28] W. Zhang, P. Mekarski, and K. Ungar, "Beta-gamma coincidence counting efficiency and energy resolution optimization by Geant 4 Monte Carlo simulations for a phoswich well detector," Applied Radiation and Isotopes, vol. 68, no. 12, pp. 2377-2381, 2010. 

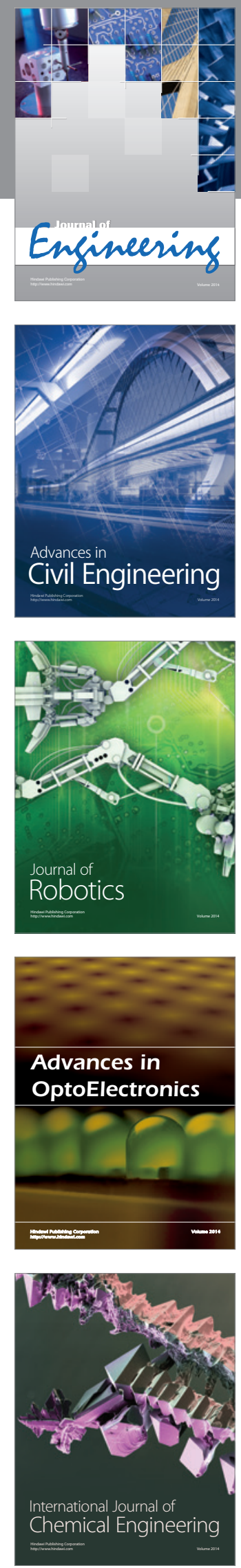

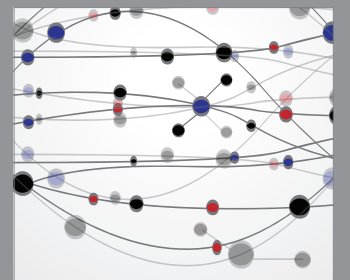

The Scientific World Journal
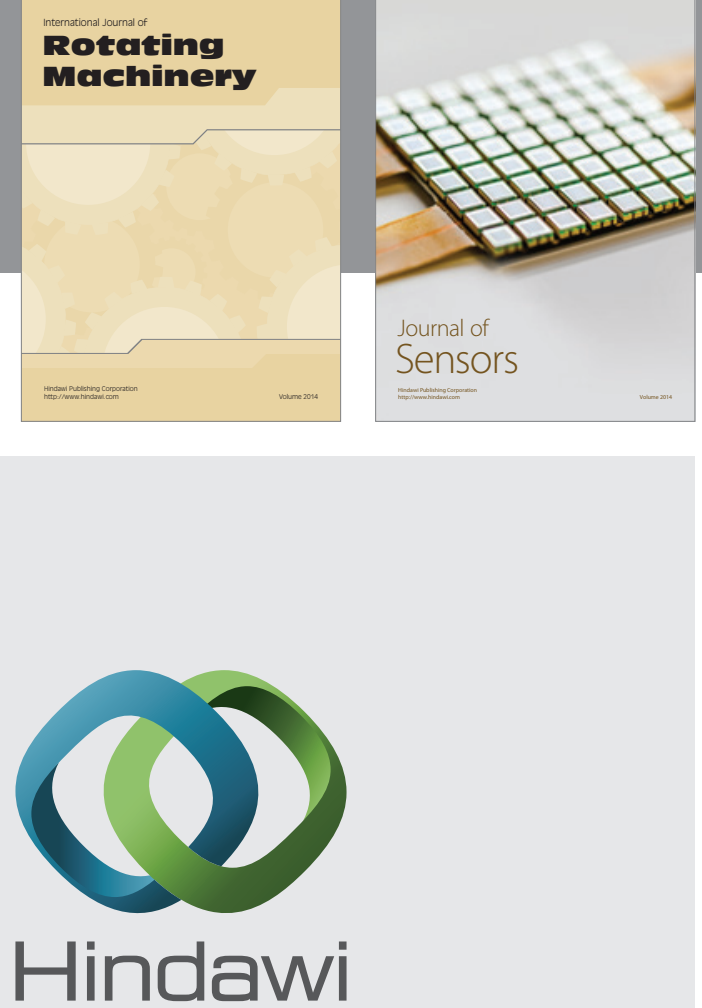

Submit your manuscripts at http://www.hindawi.com
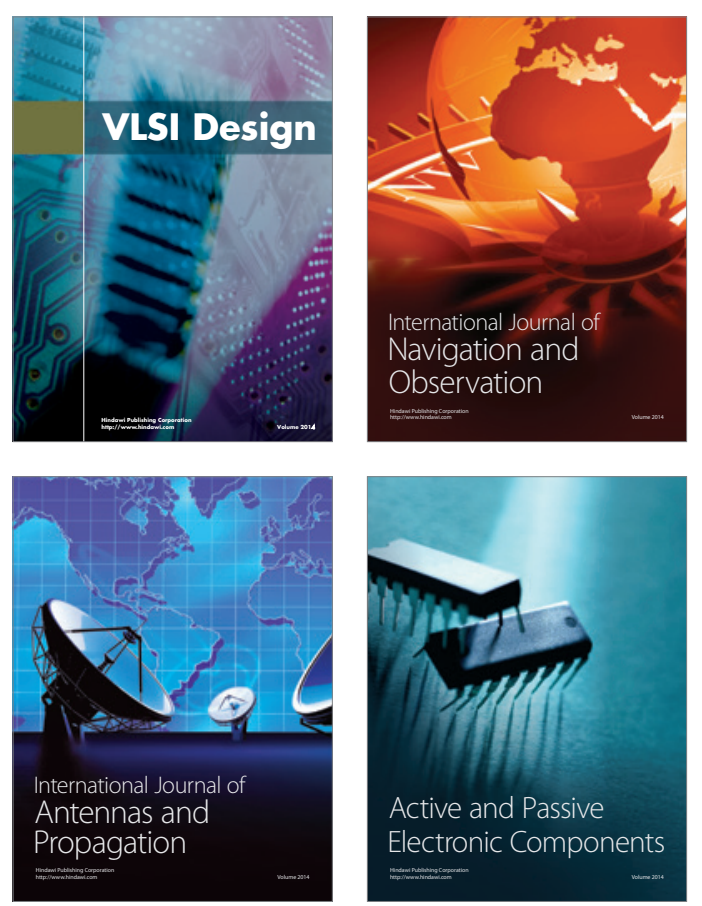
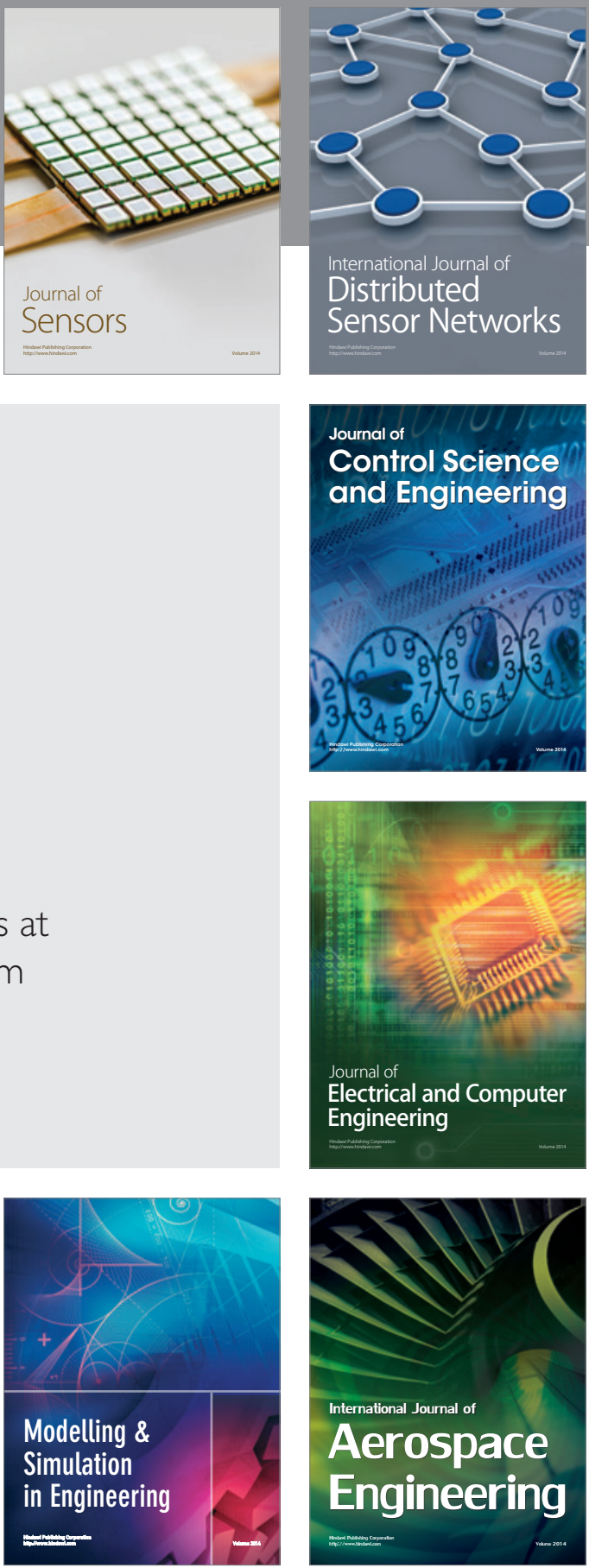

Journal of

Control Science

and Engineering
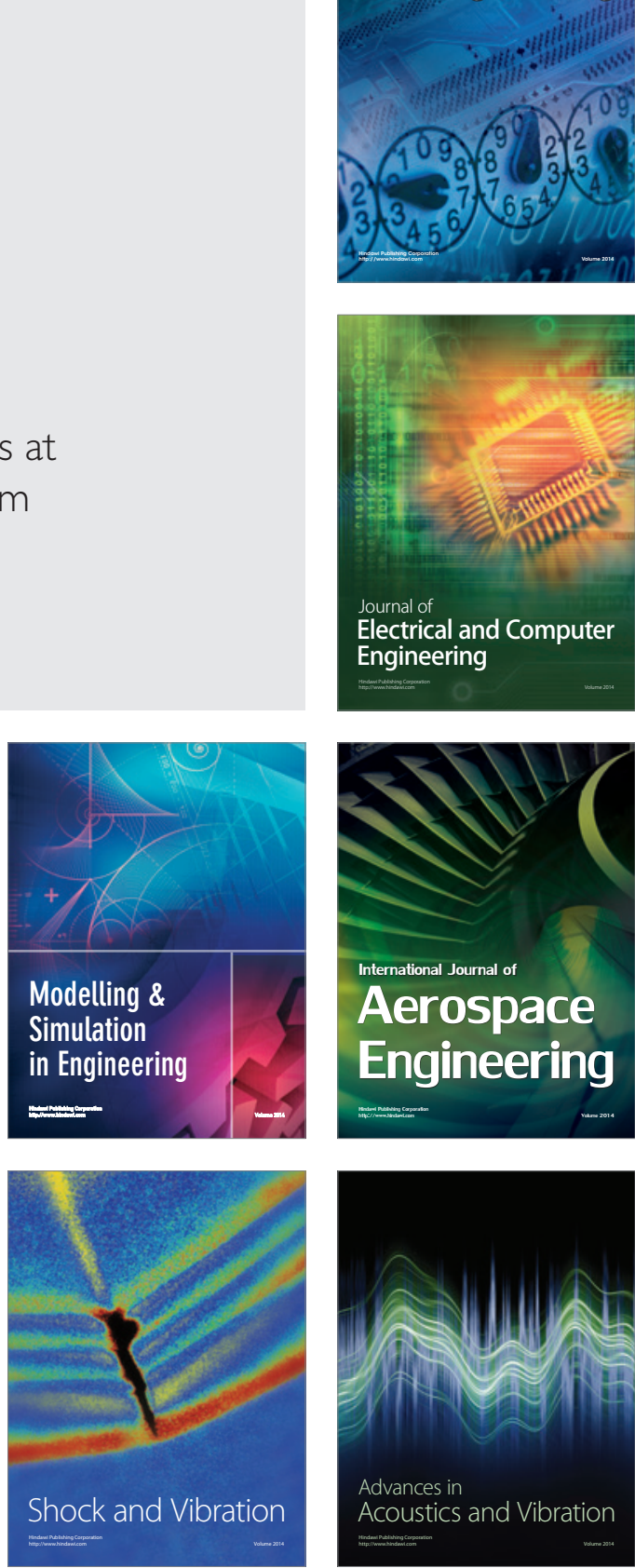